\title{
Immunohistochemical mismatch repair proteins expression as a tool to predict the melanoma immunotherapy response
}

\author{
GIOVANNI PONTI ${ }^{1}$, GIOVANNI PELLACANI ${ }^{2}$, ALDO TOMASI $^{1}$, ROBERTA DEPENNI ${ }^{3}$, \\ MONIA MACCAFERRI $^{1}$, ANTONIO MAIORANA ${ }^{4}$, GIULIA ORSI $^{3}$, \\ FRANCESCA GIUSTI $^{2}$, STEFANO CASCINU ${ }^{3}$ and MARCO MANFREDINI ${ }^{2}$
}

\begin{abstract}
Divisions of ${ }^{1}$ Clinical Pathology and ${ }^{2}$ Dermatology, Department of Surgical, Medical, Dental and Morphological Sciences with Interest in Transplant, Oncological and Regenerative Medicine; Departments of ${ }^{3}$ Oncology and Haematology, and ${ }^{4}$ Pathology, University of Modena and Reggio Emilia, I-41124 Modena, Italy
\end{abstract}

Received April 8, 2019; Accepted July 12, 2019

DOI: $10.3892 / \mathrm{mco} .2019 .1946$

\begin{abstract}
In difference to other solid malignancies, the identification of biomarkers for the prediction of malignant melanoma (MM) response to immunotherapy is limited. The aim of the current study was to evaluate the immunohistochemical (IHC) expression of MMR proteins in a cohort of MM metastatic patients receiving anti PD-1 treatments. The therapeutic response of patients was also retrospectively assessed. The cohort of the current study included 14 patients with advanced MM that had received anti PD-1 from January 2014 to December 2016 (12 males, 2 females; average age, 71 years; age range, $47-88$ years). IHC analysis of MLH1, PMS2, MSH2 and MSH6 proteins was performed on paraffin-embedded primary tumor samples from each patient and on the 23 available metastasis specimens obtained from the Division of Pathology (University of Modena and Reggio Emilia). The results revealed that $7 \%$ of the primary melanoma tissue obtained from the patient cohort exhibited the loss of expression of at least one MMR protein. Three samples from one patient, including one primary melanoma and two metastases, exhibited no MSH6 expression and had the most successful response to anti PD-1 treatment, with a progression-free survival and overall survival of 956 and 2,546 days, respectively. In conclusion, the assessment of
\end{abstract}

Correspondence to: Dr Giovanni Ponti, Division of Clinical Pathology, Department of Surgical, Medical, Dental and Morphological Sciences with Interest in Transplant, Oncological and Regenerative Medicine, University of Modena and Reggio Emilia, 71 Via del Pozzo, I-41124 Modena, Italy

E-mail: giovanni.ponti@unimore.it

Abbreviations: MM, malignant melanoma; MMR, mismatch repair; MMR-d, mismatch repair deficiency; MMR-s, mismatch repair stable; MSI, microsatellite instability; IHC, immunoistochemistry

Key words: immunohistochemistry, immunotherapy, malignant melanoma
MMR protein expression represents a potential predictive marker that may have critical importance for patients with primary and metastatic MM, primarily as criterion for the adoption of immunotherapy treatments.

\section{Introduction}

At present, there is growing evidence suggesting that microsatellite instability (MSI) may be one possible predictive marker of immunotherapy response in many cancer types and in malignant melanoma (MM) (1-7). The main cause of MSI is a defect in the DNA mismatch repair (MMR) genes, whose function is to repair the mismatched bases (8). Several repair genes (MSH2, MSH3, MSH6, MLH1, MLH3, PMS1 and $P M S 2$ ) are involved in this process and work by forming heterodimers. The interaction between mismatch recognition complexes and other proteins such as helicase, proliferating cell nuclear antigen, replication protein $\mathrm{A}$, exonuclease 1 is required for the rectification of base-pair alterations, insertion-deletion loops and hetero-duplexes instigated during replication and recombination (9). MMR genes aberrations can be investigated by immunohistochemistry (IHC) for MMR proteins, which is a quick and simple assay to recognize MMR status (10-12).

There is a clear relationship between the loss of IHC expression of one or more MMR proteins and the MSI phenomenon $(13,14)$. It was also known that the MSI status and the lack of MMR protein expression constitute a positive prognostic factor for colon cancer patients.

The reason of this evidence has been found in the enhanced immunogenicity of tumors characterized by MSI and MMR deficiency (MMR-d), because their defective ability to repair DNA damages lead to a higher mutational burden and an increased generation of neoantigens (15).

The involvement of MMR genes was proposed in MM tumorigenesis (16). MSI was described in displastic naevi, MMs and MM metastases, suggesting that the MMR system and consequently MSI could contribute to the pathogenesis of MM (17). Differently to colon cancer and other solid malignancies that are treated with immunotherapy $(1,18,19)$, potential IHC biomarkers are still limited for MM up to 
now. This lack of predictive biomarkers for immunotherapy response in MM patients is today an important field of investigation, whose aim is to allow a personalized therapeutic approach. A targeted treatment in fact maximizes the patient's outcome, avoiding unnecessary risks for the patient's health and sparing important medical resources, because of the high costs of the new bioengineered drugs. The aim of our report was to evaluate the role of MMR IHC proteins expression as a predictive marker of immunotherapy response.

\section{Patients and methods}

The study was conducted in accordance with the World Medical Association Declaration of Helsinki regarding ethical conduct of research. The study was regularly approved on October 2014 by the ethics committee of the University of Modena and Reggio Emilia and written informed consent was provided by patients for IHC analysis. As a part of a systematic review of the medical record of patients affected by advanced MM receiving anti PD-1 treatment from 2014 to 2016 at the Dermatology and Oncology Department of the University of Modena and Reggio Emilia, we collected the following data: Clinical stage of disease (AJCC 8th Ed., stage I-IV) (20); primary tumor location; overall survival (OS), progression-free survival (PFS) after anti PD-1 therapy; adverse events during anti PD-1 therapy. PFS was determined using the RECIST criteria (21).

IHC analysis of MSH6, MLH1, MSH2 and PMS2 proteins were carried out on the paraffin-embedded primary tumor samples of every included patients and on the available metastasis samples. The sections were cut (3-4 $\mu \mathrm{m})$ into superfrost plus microscope slides and allowed to dry at $37^{\circ} \mathrm{C}$ overnight. The slides were submitted to antigen retrieval using microwave in $10 \mathrm{mmol} / \mathrm{l}$ citrate buffer, $\mathrm{pH} 6$, at $350 \mathrm{~W}$ for $30 \mathrm{~min}$. Immunoperoxidase staining, using diaminobenzidine as chromogen, was run with the NEX-ES Automatic Staining System (Ventana). Monoclonal antibodies anti-MSH6 (Clone 44; Transduction Laboratories; BD Biosciences) at 1:2,000, anti-MLH1 (G168-15) at 1:40, anti-MSH2 (G129-1129; both Pharmingen) at 1:40 dilution and anti PMS-2 (Biocare Medical) at 1:40 dilution were used. Nuclei were counterstained with hematoxylin and adjacent normal tissue in each sample served as positive control. The complete absence of staining of tumor cells for one of the MMR proteins was considered indicative of a mismatch repair defect.

\section{Results}

We were able to identify 14 patients with advanced MM that were treated with anti PD-1 during 2014-2016 (Table I). Patients comprised 12 males and 2 females, average age was 71 years, ranging from 47 to 88 years. MM was located on the head and neck area $(n=2,14 \%)$, on the trunk $(n=7,50 \%)$, on the upper $\operatorname{limb}(n=3,22 \%$, of which 2 were acral) and on the lower limb ( $n=2,14 \%$, of which 1 was acral); no patient of our series was affected by mucosal melanoma. The total number of metastases was 45: 10 were located at the lungs, 8 at the lymph nodes, 6 at the skin, 3 in the retroperitoneum,
3 at the bone, 3 at the spleen, 3 at the liver, 3 at the brain, 2 at the muscle, 1 at the gut, 1 at the stomach, 1 at the pancreas and 1 at the kidney. Of these, only 23 were available as paraffin embedded samples.

Only three samples showed MMR-d of MMR genes, in particular of MSH6: they were one primary subungual melanoma and two metastases (brain and ileus) belonging to the same female patient, whose complex clinical history is summarized by the time plot represented in Fig. 1. The IHC panels of the primary MM and related metastases of our MMR-d and of an exemplificative MMR normal patient are shown in Figs. 2 and 3, respectively. Remarkably, she showed the best response to anti PD-1 treatment of our cohort, with PFS and OS of 956 and 2,546 days, respectively. At present, the patient is still alive and in complete response. By contrast, MMR-s patients showed average PFS and OS of 290 and 542 days, respectively.

\section{Discussion}

The assessment of MMR protein expression represents a potential predictive marker which may have crucial importance for primary and metastatic MM patients. The identification of quick and simple assays to predict the response to anti PD-1 agents is one of the goal that the new era of MM immunotherapy should achieve in order to satisfy the compelling objectives of a personalized medicine.

Several studies have demonstrated that MMR gene deficiency is a more widespread phenomenon than first assumed, being present in many tumor types, such as colon cancer, ovarian cancer, brain tumors, biliary tract tumors and gastric cancer, but also in neuroblastoma and endometrial cancer (17). In a recent study by Kim et al (22) the MMR-d tumor status was assessed in 430 consecutive solid tumors and was significantly correlated to the PD-L1 expression. Even though MMR-d is a negative biomarker for MM chemotherapy sensitivity (23), recent evidences suggest that it may be a positive biomarker for immunotherapy response. In detail, it was demonstrated that MSI, which is often a consequence of MMR gene deficiency, is associated to a better response to immunotherapy in terms of PFS. As a general consideration, our hypothesis is that the presence of a mutator tumor phenotype allows an easier escape of the tumor to the pharmacokinetis of conventional chemotherapy, but, on the other hand, it produces many neoantigens that increase the immunogenicity of tumor cells. Thus, it is our hypothesis that the IHC analysis of MMR gene could be a marker that predict PFS and OS of MM patients treated by anti-PD-1 agents. In our population, the best PFS response was reached by the patient that showed MMR-d tumor status, with lack of expression of MSH6, not associated to Lynch syndrome or Muir-Torre Syndrome. The clinical history of the patient was complex and the previous treatment lead to unsatisfactory results. Noteworthy, anti PD-1 therapy lead to a dramatic improvement of her management, with the regression of the brain and other metastasis, that are generally considered the prognostic factors that most negatively affect patient's survival. The limitations of our study consisted in the small population size and the lack of molecular biology investigations. Future studies are needed to investigate the 


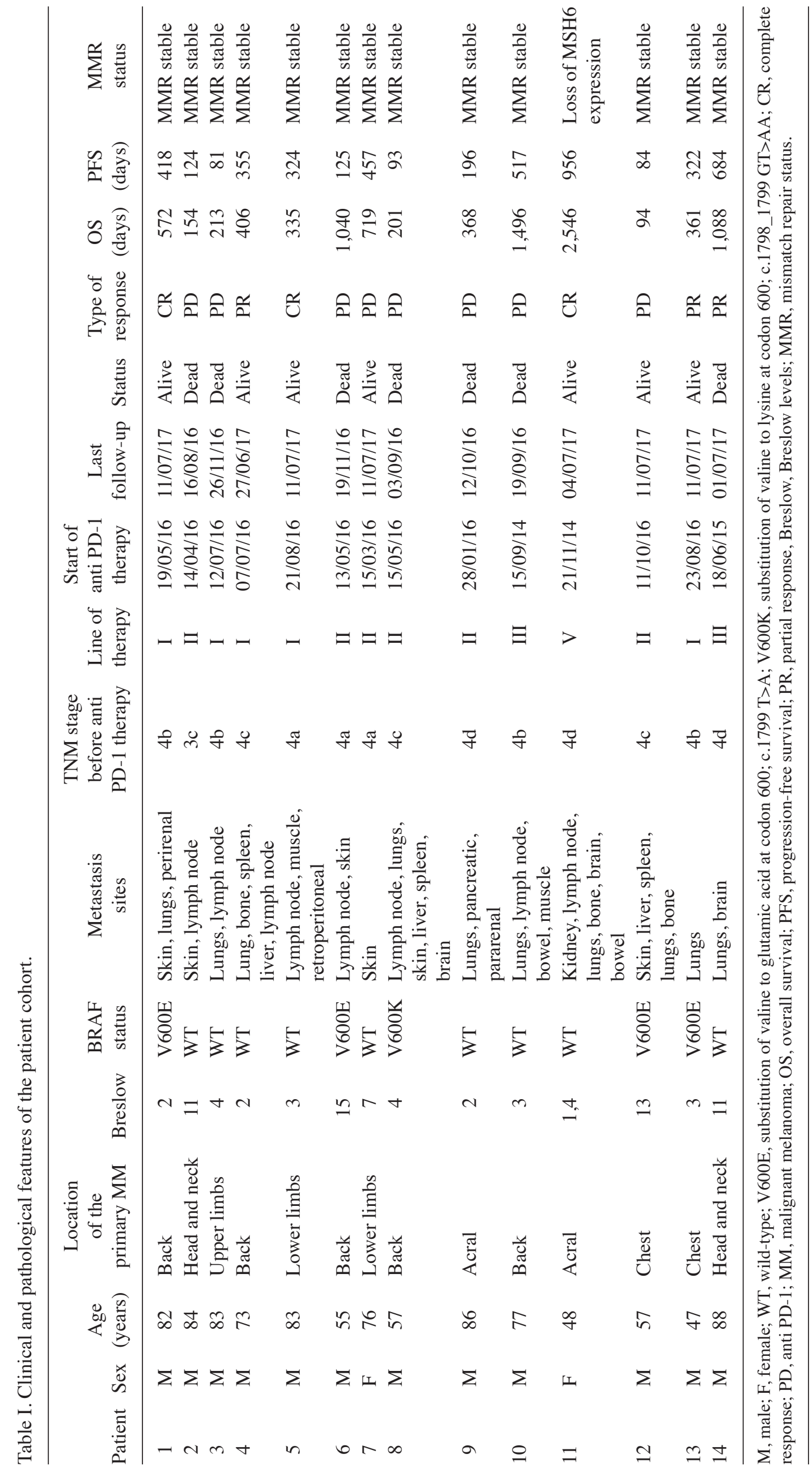



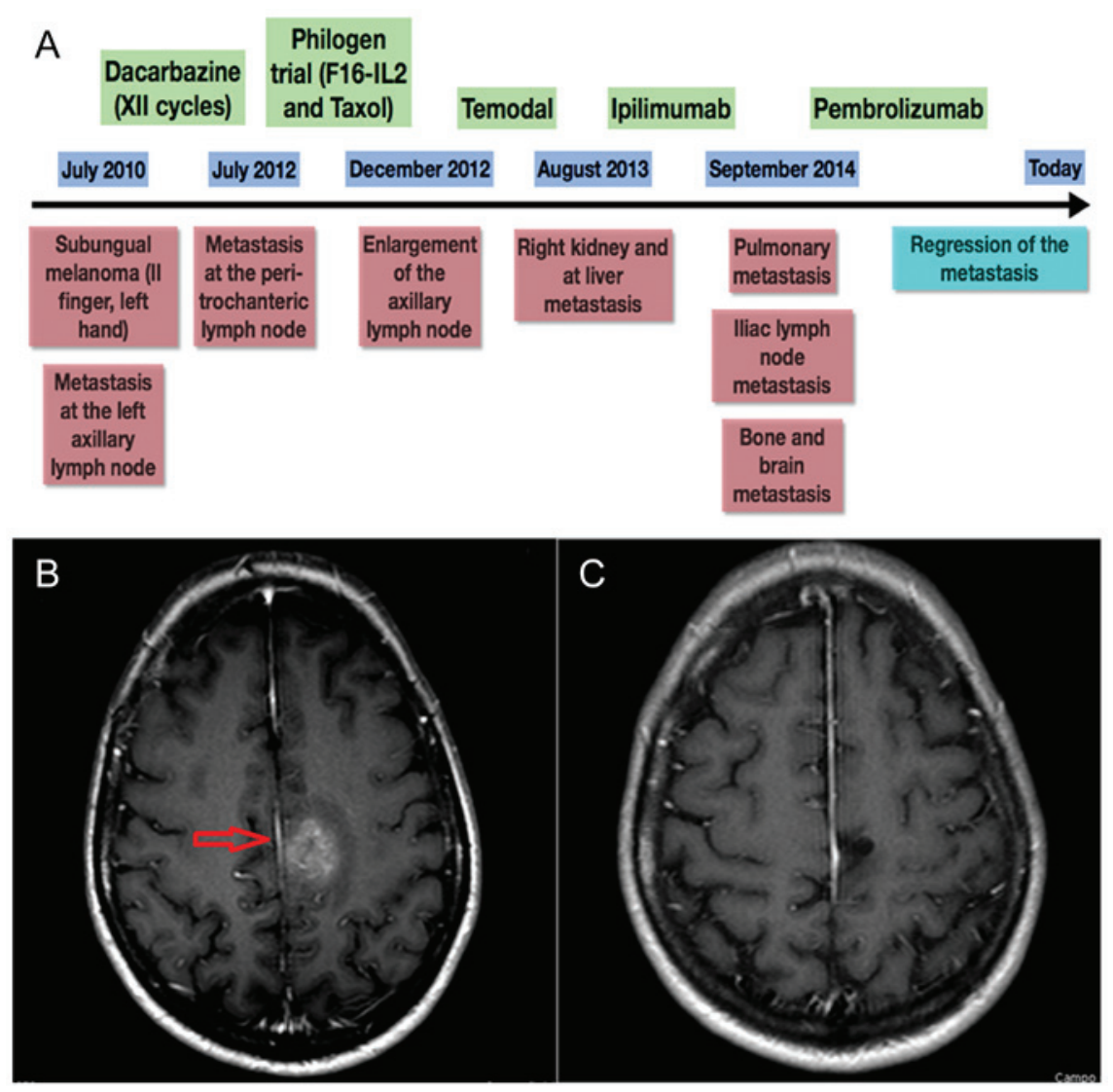

Figure 1. Patient history. (A) Clinical history schematic of a representative patient who exhibited a loss of MSH6 expression in primary subungual melanoma tissue and in ileal and brain metastasis tissue. (B) CT scan of the patient's brain, presenting metastasis that was present prior to PD-1 treatment. (C) Last CT scan of the patient, presenting regression of the metastasis (red arrow) after treatment with anti PD-1. Green boxes represent the drugs administered, blue boxes represent follow-up times and red boxes denote the associated clinical-instrumental status. MSH6, clone 44 Ventana; PD-1, programmed death ligand 1.
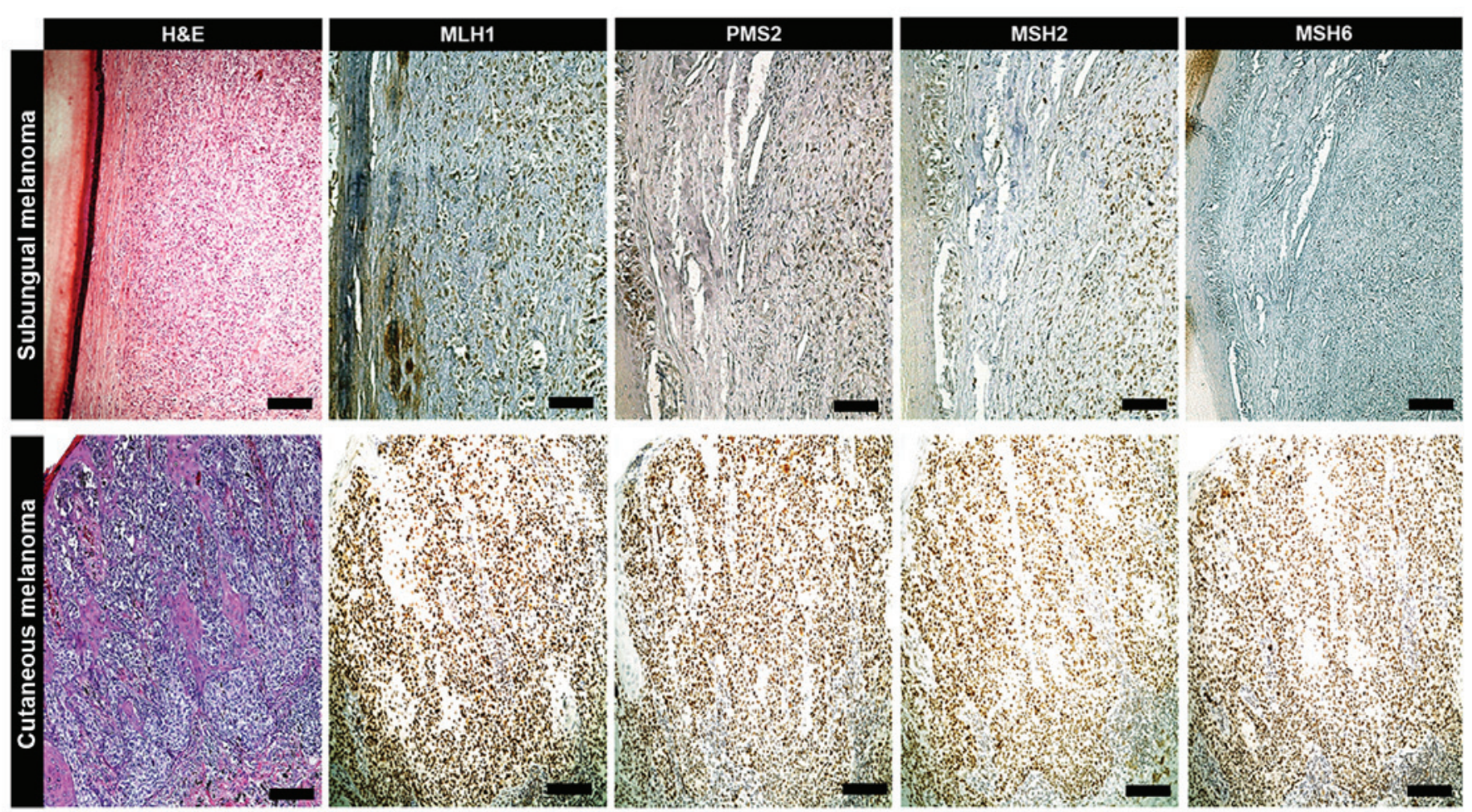

Figure 2. IHC of primary melanoma. When compared with MLH1, PMS2 and MSH2 levels, an exclusive loss of MSH6 expression was exhibited in a female case of subungual melanoma form the patient cohort, as determined via IHC. In the remaining cases of cutaneous melanoma, the expressions of MLH1, PMS2, MSH2 and MSH6 were within the normal range (scale bars, $100 \mu \mathrm{m}$ ). IHC, immunohistochemistry; MLH1, clone M1 Ventana; PMS2, clone EPR3947 Ventana; MSH2, clone G219-1129; MSH6, clone 44 Ventana; H\&E, hematoxylin and eosin. 

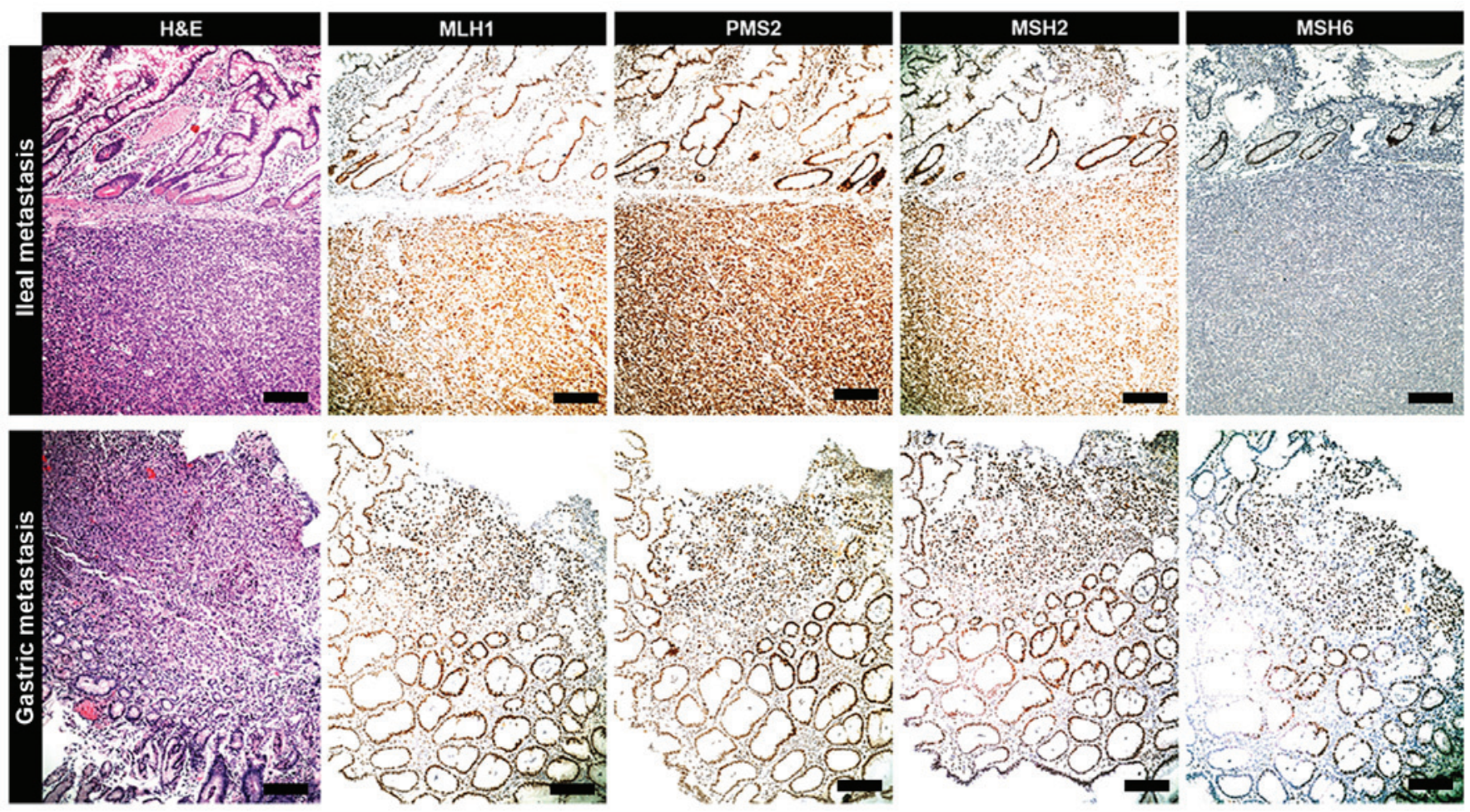

Figure 3. IHC of metastasis. Ileal metastasis tumor originating from subungual melanoma exhibits the same IHC profile of primitive neoplasia. It is characterized by an exclusive loss of expression of MSH6 protein when compared with MLH1, PMS2 and MSH2 status. In the analyzed metastases of the current cohort (e.g., gastric metastasis), the expression of MSH6 protein was preserved as with that of MLH1, PMS2 and MSH2 (scale bars, $100 \mu \mathrm{m}$ ). IHC, immunohistochemistry; MSH6, clone 44 Ventana; MLH1, clone M1 Ventana; PMS2, clone EPR3947 Ventana; MSH2, clone G219-1129; H\&E, hematoxylin and eosin.

role of MSI and MMR-d during immunotherapy in animal model as well as in larger patient cohorts.

In conclusion, the assessment of MMR protein expression represents a potential predictive marker, which may have crucial importance for primary and metastatic MM patients, mainly as criterion for the adoption of the immunotherapy treatments.

\section{Acknowledgements}

Not applicable.

\section{Funding}

No funding was received.

\section{Availability of data and materials}

All data generated or analyzed during this study are included in this published article.

\section{Authors' contributions}

GPo interpreted patient data and wrote the manuscript. GPe and AT analyzed and interpreted patient data. RD and FG enrolled and examined the patients with melanoma. MMac analyzed the clinical and molecular data. AM performed histological examinations. GO collected the clinical data and examined the specimens. SC interpreted the patient data.
MMan provided clinical advice and wrote the manuscript. All authors read and approved the final manuscript.

\section{Ethics approval and consent to participate}

The present study was approved on October 2014 by the Ethics Committee of the University of Modena and Reggio Emilia and written informed consent for participation in the study or use of their tissue was obtained from all participants.

\section{Patient consent for publication}

Written informed consent was obtained for the publication of data and materials. All data are published with respect to participants' rights to privacy and to protect their identity.

\section{Competing interests}

The authors declare that they have no competing interests.

\section{References}

1. Gargiulo P, Della Pepa C, Berardi S, Califano D, Scala S, Buonaguro L, Ciliberto G, Brauchli P and Pignata S: Tumor genotype and immune microenvironment in POLE-ultramutated and MSI-hypermutated endometrial cancers: New candidates for checkpoint blockade immunotherapy? Cancer Treat Rev 48: 61-68, 2016.

2. Dudley JC, Lin MT, Le DT and Eshleman JR: Microsatellite instability as a biomarker for PD-1 blockade. Clin Cancer Res 22: 813-820, 2016. 
3. Bupathi $\mathrm{M}$ and Wu C: Biomarkers for immune therapy in colorectal cancer: Mismatch-repair deficiency and others. J Gastrointest Oncol 7: 713-720, 2016.

4. Czink E, Kloor M, Goeppert B, Fröhling S, Uhrig S, Weber TF, Meinel J, Sutter C, Weiss KH, Schirmacher P, et al: Successful immune checkpoint blockade in a patient with advanced stage microsatellite-unstable biliary tract cancer. Cold Spring Harb Mol Case Stud 3: a001974, 2017.

5. Chen L, Xiong Y, Li J, Zheng X, Zhou Q, Turner A, Wu C, Lu B and Jiang J: PD-L1 expression promotes epithelial to mesenchymal transition in human esophageal cancer. Cell Physiol Biochem 42: 2267-2280, 2017.

6. Howitt BE, Strickland KC, Sholl LM, Rodig S, Ritterhouse LL, Chowdhury D, D'Andrea AD, Matulonis UA and Konstantinopoulos PA: Clear cell ovarian cancers with microsatellite instability: A unique subset of ovarian cancers with increased tumor-infiltrating lymphocytes and PD-1/PD-L1 expression. Oncoimmunology 6: e1277308, 2017.

7. Benatti $\mathrm{P}, \mathrm{Gafà} \mathrm{R}$, Barana D, Marino M, Scarselli A, Pedroni M, Maestri I, Guerzoni L, Roncucci L, Menigatti M, et al: Microsatellite instability and colorectal cancer prognosis. Clin Cancer Res 11: 8332-8340, 2005.

8. Richman S: Deficient mismatch repair: Read all about it (Review). Int J Oncol 47: 1189-1202, 2015.

9. Bhattacharya P and Patel TN: Microsatellite instability and promoter hypermethylation of DNA repair genes in hematologic malignancies: A forthcoming direction toward diagnostics. Hematology 23: 77-82, 2018.

10. Chapusot C, Martin L, Puig PL, Ponnelle T, Cheynel N, Bouvier AM, Rageot D, Roignot P, Rat P, Faivre J and Piard F: What is the best way to assess microsatellite instability status in colorectal cancer? Study on a population base of 462 colorectal cancers. Am J Surg Pathol 28: 1553-1559, 2004.

11. Ponti G, Losi L, Di Gregorio C, Roncucci L, Pedroni M, Scarselli A, Benatti P, Seidenari S, Pellacani G, Lembo L, et al: Identification of Muir-Torre syndrome among patients with sebaceous tumors and keratoacanthomas: Role of clinical features, microsatellite instability, and immunohistochemistry. Cancer 103: 1018-1025, 2005.

12. Ponti G, Losi L, Pedroni M, Lucci-Cordisco E, Di Gregorio C, Pellacani G and Seidenari S: Value of MLH1 and MSH2 mutations in the appearance of Muir-Torre syndrome phenotype in HNPCC patients presenting sebaceous gland tumors or keratoacanthomas. J Invest Dermatol 126: 2302-2307, 2006

13. Garcia JJ, Kramer MJ, O'Donnell RJ and Horvai AE: Mismatch repair protein expression and microsatellite instability: A comparison of clear cell sarcoma of soft parts and metastatic melanoma. Mod Pathol 19: 950-957, 2006.

14. Ponti G and Longo C: Microsatellite instability and mismatch repair protein expression in sebaceous tumors, keratocanthoma, and basal cell carcinomas with sebaceous differentiation in Muir-Torre syndrome. J Am Acad Dermatol 68: 509-510, 2013.
15. Nebot-Bral L, Coutzac C, Kannouche PL and Chaput N: Why is immunotherapy effective (or not) in patients with MSI/MMRD tumors? Bull Cancer 106: 105-113, 2019.

16. Alvino E, Marra G, Pagani E, Falcinelli S, Pepponi R, Perrera C, Haider R, Castiglia D, Ferranti G, Bonmassar E, et al: High-frequency microsatellite instability is associated with defective DNA mismatch repair in human melanoma. J Invest Dermatol 118: 79-86, 2002.

17. Ponti G, Losi L, Pellacani G, Wannesson L, Cesinaro AM, Venesio T, Petti C and Seidenari S: Malignant melanoma in patients with hereditary nonpolyposis colorectal cancer. $\mathrm{Br}$ J Dermatol 159: 162-168, 2008.

18. Ponti G, Manfredini M, Greco S, Pellacani G, Depenni R, Tomasi A, Maccaferri M and Cascinu S: BRAF, NRAS and C-KIT advanced melanoma: Clinico-pathological features, targeted-therapy strategies and survival. Anticancer Res 37: 7043-7048, 2017

19. Moscarella E, Pellegrini C, Pampena R, Argenziano G, Manfredini M, Martorelli C, Ciarrocchi A, Dika E, Peris K, Antonini A, et al: Dermoscopic similarity is an independent predictor of BRAF mutational concordance in multiple melanomas. Exp Dermatol 28: 829-835, 2019.

20. Amin MB, Edge S, Greene F, Byrd DR, Brookland RK, Washington MK, Gershenwald JE, Compton C, Hess KR, Sullivan DC, et al (eds.): Cancer Staging Manual. 8th edition. Springer, New York, NY, 2017.

21. Eisenhauer EA, Therasse P, Bogaerts J, Schwartz LH, Sargent D, Ford R, Dancey J, Arbuck S, Gwyther S, Mooney M, et al: New response evaluation criteria in solid tumours: Revised RECIST guideline (version 1.1). Eur J Cancer 45: 228-247, 2009.

22. Kim ST, Klempner SJ, Park SH, Park JO, Park YS, Lim HY, Kang WK, Kim KM and Lee J: Correlating programmed death ligand 1 (PD-L1) expression, mismatch repair deficiency, and outcomes across tumor types: Implications for immunotherapy. Oncotarget 8: 77415-77423, 2017.

23. Lage H, Christmann M, Kern MA, Dietel M, Pick M, Kaina B and Schadendorf D: Expression of DNA repair proteins hMSH2, hMSH6, hMLH1, O6-methylguanine-DNA methyltransferase and N-methylpurine-DNA glycosylase in melanoma cells with acquired drug resistance. Int J Cancer 80: 744-750, 1999.

This work is licensed under a Creative Commons Attribution-NonCommercial-NoDerivatives 4.0 International (CC BY-NC-ND 4.0) License. 